metrischer Form durch galvanische Überzüge. (Metalloberfläche 15, 357-361, 1961.)

Die Einebnung durch galvanische Überzüge wird auf einfache Weise rechnerisch behandelt.

Zur Berechnung der Einebnung wird folgende Formel vorgeschlagen:

$$
E=\frac{d_{2}-d_{1}}{t} \cdot 100 \text { in } \% \text {. }
$$

Die damit erhaltenen Werte sind anschaulich und für Vergleichszwecke geeignet.

Für die geometrische Einebnung gelten einfache geometrische Beziehungen. Die geometrische Einebnung gehorcht der Formel:

$$
E_{g}=\frac{d_{1}\left(1-\sin \frac{a}{2}\right)}{t \cdot \sin \frac{a}{2}} \cdot 100 \text { in } \% \text {. }
$$

Diese Formel hat allerdings nur dann Gültig. keit, wenn der Überzug an allen Stellen einer Kerbe gleich schnell wächst, d. h. wenn eine ideale Mikrostreufähigkeit vorliegt. Diese Forderung ist bei Kupfer- und Nickelniederschlägen aus sauren Bädern unter bestimmten Abscheidungsbedingungen nahezu erfüllt.

Die echte Einebnung hängt von einer Vielzahl von Faktoren ab. Für sie läßt sich nur unter voreinfachenden Annahmen eine Näherungsformel aufstellen. Die Schwierigkeiten bei der mathematischen Behandlung der echten Einebnung werden noch dadurch erhöht, daß echte Einebnung stets mit geometrischer Einebnung gekoppelt ist. Beide beeinflussen sich wechselseitig.

Auf die scheinbare Einebnung durch Bildung maskierter Poren wird hingewiesen.

$$
\vec{F} \text {. Peters (Hagen/W.) }
$$

\section{Anstriche, Lacke, Harze, Wachse}

Črmáková, D. (Staat. Forschungsinst. für Materialschutz G. V. Akimov, Praha, CSSR). Sorption und Quellung der Anstrichfilme. (Chemický prưmysl 11 (36), 497-500, 1961.)

Die Untersuchung der Sorption und der Quellung der Anstrichfilme (namentlich der Alkydfilme) mittels der gravimetrischen und mikroskopischen Methoden ergänzt durch eine qualitative Wertung ermöglichte, den Mechanismus dieser Vorgänge bei chemisch sowie physikalisch trocknenden Filmen zu erörtern. Destillierwasser, $10 \%$ ige Salzsäure und $0,1 \mathrm{~N}$ wäßrige Lösung von $\mathrm{NaOH}$ wurden als korrosive Media benützt. Bei den chemisch trocknenden Filmen wurde der verlangsamende Einfluß der Grenzflächen zwischen den einzelnen Schichten festgestellt, der das Durchdringen von Wasser und Ionen verhindert. Gleichzeitig sind aber diese Flächen auch Stellen der Bildung von osmotischen Blasen zwischen den Schichten. Die in der Arbeit angeführte Gesetzmäßigkeit gestattet, einige Defekte der Anstrichfilme bei praktischer Benützung zu erklären.

\title{
Berichtigung
}

\section{zur Arbeit \\ Diffusion-Controlled Transition in High Polymers

\author{
By K. Kanamaru und M. Sugiura (Tokyo)
} \\ Kolloid-Z. 178, 1-24 (1961)}

Infolge Zeitmangels ist es nicht möglich gewesen die Autorkorrekturen zu dieser Arbeit rechtzeitig zu berücksichtigen.

Es sind daher folgende Verbesserungen nach zutragen :

S. 3 linke Spalte, Gleichung 3 :

$$
\begin{aligned}
& \text { Lies }\left(Q_{m}\right)_{a}=\frac{\left(Q_{m}\right)_{90}{ }^{\circ} \mathrm{C}}{1-\left(X_{c}\right)_{90}{ }^{\circ} \mathrm{C}} \\
& \text { statt }\left(Q_{m}\right)_{a}=\frac{\left(Q_{m}\right)_{90}{ }^{\circ} \mathrm{C}}{\mathrm{I}-\left(X_{c}\right)_{90}{ }^{\circ} \mathrm{C}} \cdot \frac{(\varrho)_{90}{ }^{\circ} \mathrm{C}}{\left(\varrho_{a}\right)_{90^{\circ} \mathrm{C}}} .
\end{aligned}
$$

S. 3 linke Spalte, Zeile 9: der hinter ,,where" und vor ,$\left(X_{C}\right)_{90}{ }^{\circ} \mathrm{C}$ " stehende Wortlaut ist $\mathrm{zu}$ streichen. Also soll Zeile 9 bis 11 heißen,, where $\left(X_{c}\right)_{90}{ }^{\circ} \mathrm{C}$ is the crystalline fraction of polyethylene also at $90^{\circ} \mathrm{C}^{64}$.

S. 3 rechte Spalte, Gleichung in Zeile 8 (von oben): Lies $Q_{m}=\left(Q_{m}\right)_{a} \cdot\left(1-X_{c}\right)$

statt $Q_{m}=\left(Q_{m}\right)_{a} \cdot\left(1-\bar{X}_{c}\right) \cdot \varrho_{a} \varrho \varrho$

$$
=\left(Q_{m}\right)_{a}\left(1-X_{c}\right) \cdot V / V_{a} \text {. }
$$

S. 3 rechte Spalte, Zeile 2 (von oben): Lies 0.0933 statt 0.110 .

S. 3 rechte Spalte, Zeile 3 (von oben): Lies 0.107 statt 0.115 .
S. 4 Tabelle 1, die Überschrift von Spalte 5: Lies $X_{c}($ Wt. $-\%)$ statt $X_{c}($ Vol.- \%).

S. 4 Tabelle 1, Spalte 3 :

Lies $\left\{\begin{array}{l}0.801 \\ 0.801 \\ 0.799 \\ 0.799 \\ 0.796 \\ 0.796\end{array}\right.$ statt $\left\{\begin{array}{l}0.799 \\ 0.799 \\ 0.796 \\ 0.796 \\ 0.792 \\ 0.792\end{array}\right.$

S. 4 Tabelle 1, Spalte 5:

Lies $\left\{\begin{array}{l}35.8 \\ 35.8 \\ 31.8 \\ 31.8 \\ 24.9 \\ 24.9\end{array}\right.$ statt $\left\{\begin{array}{l}37.4 \\ 37.4 \\ 32.8 \\ 32.8 \\ 26.8 \\ 26.8\end{array}\right.$

S. 18 linke Spalte, die Unterschrift der Fig. 15: Lies $\log \tau$ vs $1 / T\left({ }^{\circ} \mathrm{K}\right)$ statt $\log D$ vs $1 / T\left({ }^{\circ} \mathrm{K}\right.$.

Die auf der Stelle von Fig. 9 angegebene Abbildung (auf S. 10) und die auf der Stelle von Fig. 15 angegebene Abbildung (auf S. 18) sind auszuwechseln.

Für die Schriftleitung verantwortlich: Für Originalarbeiten Prof. Dr. F. H. Müller, 3550 Marburg/Lahn und für Referate und Berichte Dr. E. Ưhlein, 6000 Frankfurt/M.

Anzeigenverwaltung: Dr. Karl Niedermeyer, 6000 Frankfurt/M.-West, Georg-Speyer-Straße 76

Dr. Dietrich Steinkopff, Verlag 6100 Darmstadt, Saalbaustraße 12 Satz und Druck: Universitätsdruckerei Mainz GmbH 